\title{
Prudent Semantics for Argumentation Frameworks
}

\author{
Sylvie Coste-Marquis, Caroline Devred and Pierre Marquis* \\ CRIL/CNRS - Université d'Artois — Lens - FRANCE \\ \{coste,devred,marquis\}@ cril.univ-artois.fr
}

\begin{abstract}
We present new prudent semantics within Dung's theory of argumentation. Under such prudent semantics, two arguments cannot belong to the same extension whenever one of them attacks indirectly the other one. We argue that our semantics lead to a better handling of controversial arguments than Dung's ones. We compare the prudent inference relations induced by our semantics w.r.t. cautiousness; we also compare them with the inference relations induced by Dung's semantics.
\end{abstract}

\section{Introduction}

Argumentation is a general approach to model defeasible reasoning, in which the two main issues are the generation of arguments and their exploitation so as to draw some conclusions based on the way arguments interact (see e.g., $[17,15])$.

Several theories of argumentation have been proposed so far (see among others $[10,14,16,12,4,1,7])$. Among them is Dung's theory ${ }^{1}[10]$, which is quite influential since it encompasses many approaches to nonmonotonic reasoning and logic programming as special cases. In Dung's approach, no assumption is made about the nature of an argument (it can be a statement supported by some assumptions like in the theory introduced by Elvang-Gøransson et al. [13] but this is not mandatory). What really means is the way arguments interact w.r.t. the attack relation. Arguments and the way they interact w.r.t. the attack relation are considered as initial data of any argumentation framework, which can thus be viewed as a labeled digraph.

Several inference relations can be defined within Dung's theory. Usually, inference is defined at the argument level: an argument is considered derivable from an argumentation framework $A F$ when it belongs to one (credulous accept-

* The authors have been partly supported by the IUT de Lens, the Région Nord/Pas-de-Calais through the IRCICA Consortium and by the European Community FEDER Program.

${ }^{1}$ Refined and extended by several authors, including [3, 2]. ability) (resp. all (skeptical acceptability)) extension(s) of $A F$ under some semantics, where an extension of $A F$ is a conflict-free and self-defending set of arguments, maximal for a given criterion (made precise by the semantics under consideration). While skeptical acceptability can be safely extended to the level of sets of arguments, this is not the case for credulous acceptability. Indeed, it can be the case that both arguments $a$ and $b$ are (individually) derivable from $A F$ while the set $\{a, b\}$ is not included into any extension of $A F$. Now, defining acceptability for sets of arguments as inclusion into some (resp. all) extensions under Dung's semantics does not always lead to expected conclusions. Consider Example 1:

Example 1 Let $A F_{1}=\langle A, R\rangle$ with $A=\{a, b, c, e, n, i\}$ and $R=\{(b, a),(c, a),(n, c),(i, b),(e, c),(i, e)\}$. The digraph for $A F_{1}$ is depicted on the following figure.

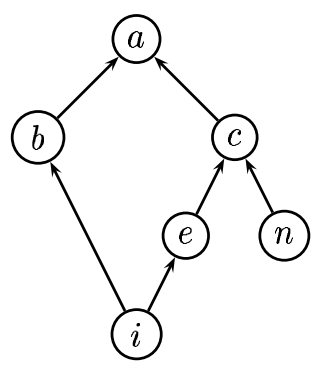

On this example, whatever the semantics and the inference relation (skeptical or credulous) among Dung's ones, $a$ is derivable from $A F_{1}$. However, $a$ is attacked by $b$ and the unique defeater of $b$ is controversial w.r.t. $a$ (an argument $i$ is said to be controversial w.r.t. an argument $a$ if $i$ indirectly attacks $a$ and $i$ indirectly defends $a$ ). So it is not cautious to infer $a$.

One way to cope with this problem is to suppress indirect conflicts ${ }^{2}$ from the different extensions (under all semantics). Forbidding indirect conflicts in extensions does not prevent all arguments which are indirectly attacked from belonging to some extensions. This only prevents pairs of

\footnotetext{
${ }^{2}$ There is an indirect conflict between two arguments when one of them attacks indirectly the other one.
} 
arguments which conflict indirectly from belonging to the same extension. In our opinion, such a cautious approach is sensible whenever we want to infer sets of arguments: infering together two arguments which conflict indirectly is not prudent.

This problem is not avoided by Dung's semantics. Thus, $A F_{1}$ has a single extension $\{i, n, a\}$ whatever the semantics among Dung's ones. Consequently, $a$ and $i$ are always jointly derivable from $A F_{1}$ while $i$ is controversial w.r.t. $a$.

In this paper, we define and study new semantics for Dung's framework, based on a more demanding notion of absence of conflict, since indirect attacks are not allowed within a prudent, admissible set. Especially, a prudent, admissible set never includes pairs of controversial arguments. We compare the inference relations induced by such new semantics with Dung's ones and show that in many cases one obtains more cautious notions of derivability.

\section{Dung's Theory of Argumentation}

Let us present some basic definitions at work in Dung's theory of argumentation [10]. We restrict them to finite argumentation frameworks.

Definition 1 (argumentation frameworks) $A$ finite argumentation framework is a pair $A F=\langle A, R\rangle$ where $A$ is a finite set of so-called arguments and $R$ is a binary relation over $A$ (a subset of $A \times A$ ), the attacks relation.

A first important notion is the notion of acceptability: an argument $a$ is acceptable w.r.t. a set of arguments whenever it is defended by the set, i.e., every argument which attacks $a$ is attacked by an element of the set.

Definition 2 (acceptable sets) Let $A F=\langle A, R\rangle$ be a finite argumentation framework. An argument $a \in A$ is acceptable w.r.t. a subset $S$ of $A$ if and only if for every $b \in A$ s.t. $(b, a) \in R$, there exists $c \in S$ s.t. $(c, b) \in R$. A set of arguments is acceptable w.r.t. $S$ when each of its elements is acceptable w.r.t. S.

A second important notion is the notion of absence of conflicts. Intuitively, two arguments should not be considered together whenever one of them attacks the other one.

Definition 3 (conflict-free sets) Let $A F=\langle A, R\rangle$ be a finite argumentation framework. A subset $S$ of $A$ is conflictfree if and only if for every $a, b \in S$, we have $(a, b) \notin R$.

Requiring the absence of conflicts and the form of autonomy captured by self-acceptability leads to the notion of admissible set.
Definition 4 (admissible sets) Let $A F=\langle A, R\rangle$ be a finite argumentation framework. A subset $S$ of $A$ is admissible for $A F$ if and only if $S$ is conflict-free and acceptable w.r.t. $S$.

The significance of the concept of admissible sets is reflected by the fact that every extension of an argumentation framework under the standard semantics introduced by Dung (preferred, stable, complete and grounded extensions) is an admissible set, satisfying some form of optimality:

Definition 5 (extensions) Let $A F=\langle A, R\rangle$ be a finite argumentation framework.

- A subset $S$ of $A$ is a preferred extension of $A F$ if and only if it is maximal w.r.t. $\subseteq$ among the set of admissible sets for $A F$.

- A subset $S$ of $A$ is a stable extension of $A F$ if and only if it is conflict-free for $A F$ and for every argument $a$ from $A \backslash S$, there exists $b \in S$ s.t. $(b, a) \in R$.

- A subset $S$ of $A$ is a complete extension of $A F$ if and only if it is admissible for AF and it coincides with the set of arguments acceptable w.r.t. itself.

- A subset $S$ of $A$ is the grounded extension of $A F$ if and only if it is the least element w.r.t. $\subseteq$ among the complete extensions of $A F$.

Example 1 (cont'ed) Let $E=\{a, i, n\}$. E is the grounded extension of $A F_{1}$, the unique preferred extension of $A F_{1}$, the unique stable extension of $A F_{1}$ and the unique complete extension of $A F_{1}$.

Formally, the complete extensions of $A F$ can be characterized as the fixed points of its characteristic function $\mathcal{F}_{A F}$ :

Definition 6 (characteristic functions) The characteristic function, denoted $\mathcal{F}_{A F}$, of an argumentation framework $A F=\langle A, R\rangle$ is defined as follows:

$\mathcal{F}_{A F}: 2^{A} \rightarrow 2^{A}$

$\mathcal{F}_{A F}(S)=\{a \mid$ a is acceptable w.r.t. $S\}$.

Among the fixed points of $\mathcal{F}_{A F}$, the grounded extension of $A F$ is the least element w.r.t. $\subseteq$ [10].

Finally, several notions of derivability of an argument (or more generally a set of arguments) from an argumentation framework $A F$ can be defined by requiring that the (set of) argument(s) is included into an extension (credulous acceptability) or every extension (skeptical acceptability) of $A F$ of a specific kind. Obviously enough, credulous derivability and skeptical derivability w.r.t. the grounded extension coincide, since there cannot be more than one grounded extension for any argumentation framework. 


\section{Prudent Extensions}

In order to address scenarios like in Example 1 in a more satisfying way, we need to refine Dung's notion of admissibility, by requiring that no indirect conflict occurs within an admissible set of arguments; this leads to the notion of p-admissible set:

Definition 7 (p-admissible sets) Let $A F=\langle A, R\rangle$ be a finite argumentation framework. $S \subseteq A$ is p(rudent)admissible for $A F$ if and only if every $a \in S$ is acceptable w.r.t. $S$ and $S$ is without indirect conflicts, i.e., there is no pair of arguments $a$ and $b$ of $S$ s.t. there is an odd-length path from a to $b$ in $A F$.

Example 1 (cont'ed) $\{i, n\}$ and its subsets are the $p$ admissible sets for $A F_{1}$.

From this definition, the next lemma follows immmediately:

Lemma 1 Let $a, b$ be two arguments of a finite argumentation framework $A F$. If a is controversial w.r.t. $b$, then $\{a, b\}$ cannot be included into any $p$-admissible set for $A F$.

Note that this lemma does not prevent $a$ or $b$ from belonging to a p-admissible set for $A F$, but not to the same one.

Actually, the absence of controversial arguments is only necessary. In particular, no arguments belonging to an oddlength cycle of $A F$ can also belong to a p-admissible set. Thus, our approach departs from [2] who consider that oddlength and even-length cycles in an argumentation framework should be considered in the same way. Especially, it is not cautious to consider within a single extension the arguments of an odd-length cycle since they attack themselves indirectly. Furthermore, any argument from an odd-length cycle is controversial w.r.t. an argument of the cycle.

On this ground, one can define several notions of prudent extensions, echoing Dung's ones. Let start with preferred $p$ extensions:

Definition 8 (preferred p-extensions) Let $A F=\langle A, R\rangle$ be a finite argumentation framework. A p-admissible set $S \subseteq A$ for $A F$ is a preferred p-extension of $A F$ if and only if $\nexists S^{\prime} \subseteq A$ s.t. $S \subset S^{\prime}$ and $S^{\prime}$ is p-admissible for $A F$.

Example 1 (cont'ed) $\{i, n\}$ is the unique preferred $p$ extension of $A F_{1}$.

We have the following proposition:

Proposition 1 Let $A F=\langle A, R\rangle$ be a finite argumentation framework.

1. The set of all p-admissible subsets of $A$ for $A F$ is a complete set of $\left(2^{A}, \subseteq\right)$.
2. For every $p$-admissble set $S \subseteq A$ for $A F$, there exists at least one preferred p-extension $E \subseteq A$ of $A F$ s.t. $S \subseteq E$.

Since $\emptyset$ is p-admissible for any $A F$, we obtain:

Corollary 1 Every finite argumentation framework $A F=\langle A, R\rangle$ has a preferred p-extension.

What can be found in preferred p-extensions? Though every argument which is not attacked belongs at least to one preferred p-extension of $A F$, it is not the case in general that it belongs to every preferred $\mathrm{p}$-extension of $A F$. On the other hand, whenever an argument belongs to a preferred $\mathrm{p}$ extension of $A F$, all its mandatory defenders also belong to it (but the condition is not sufficient):

Proposition 2 Let $A F=\langle A, R\rangle$ be a finite argumentation framework. Let $E$ be a preferred p-extension of $A F$ and $a \in A . a \in E$ only if all the mandatory defenders of a belong to $E$ as well, where $b \in A$ is a mandatory defender of a iff there exists $c \in A$ s.t. $(c, a) \in R$ and $a$ is not defended against $c$ by an element of $\{d \in A \mid d$ is a direct defender of $a$ and $d$ does not attack indirectly $a\} \backslash\{b\}$.

Example 1 (cont'ed) $a$ is not derivable (even credulously) w.r.t. our prudent semantics while it is skeptically derivable w.r.t. Dung's ones (which coincide here). The rationale for it is as follows: while $i$ is a mandatory defender of a, it also attacks it indirectly, i.e. $i$ is controversial w.r.t. a. So a cannot be accepted.

Let us now consider the notion of stable p-extension:

Definition 9 (stable p-extensions) Let $A F=\langle A, R\rangle$ be a finite argumentation framework. A subset $S$ of $A$ without indirect conflicts is a stable p-extension of $A F$ iff $S$ attacks (in a direct way) every argument from $A \backslash S$.

Example 1 (cont'ed) $A F_{1}$ has no stable p-extension.

As for Dung's extensions, we have:

Lemma 2 Every stable p-extension of a finite argumentation framework $A F$ also is a preferred p-extension of $A F$. The converse does not hold.

Let us now explain how p-extensions can be characterized using some fixed point construction:

Definition 10 (p-characteristic functions) The p-characteristic function of a finite argumentation framework $A F=\langle A, R\rangle$ is defined as follows:

$\mathcal{F}_{A F}^{p}: 2^{A} \longrightarrow 2^{A}$

$\mathcal{F}_{A F}^{p}(S)=\{a \mid$ a is acceptable w.r.t. $S$ and $S \cup\{a\}$ is without indirect conflict $\}$. 
Contrariwise to $\mathcal{F}_{A F}, \mathcal{F}_{A F}^{p}$ is in general nonmonotonic w.r.t. $\subseteq$ (and this is also the case of its restriction to the set of all p-admissible subsets of $A$ ). This prevents us from defining a notion of grounded p-extension as the least fixed point of $\mathcal{F}_{A F}^{p}$. Nevertheless:

Lemma 3 Let $A F=\langle A, R\rangle$ be a finite argumentation framework. The sequence $\left(\mathcal{F}_{A F}^{p}(\emptyset)\right)_{i \in \mathbb{N}}$ is monotonic w.r.t. $\subseteq$, and each element of it is a p-admissible set of $A F$.

Since $A$ is finite, the sequence $\left(\mathcal{F}_{A F}^{p}(\emptyset)\right)_{i \in \mathbb{N}}$ is stationary from some rank $j$, so the following definition of the grounded p-extension of $A F$ is well-founded:

Definition 11 (grounded p-extensions) Let $A F=\langle A, R\rangle$ be a finite argumentation framework. Let $j$ be the lowest integer such that the sequence $\left(\mathcal{F}_{A F}^{p}(\emptyset)\right)_{i \in \mathbb{N}}$ is stationary from rank $j . \mathcal{F}_{A F}^{p, j}(\emptyset)$ is the grounded p-extension of $A F$.

Example 1 (cont'ed) $\{i, n\}$ is the grounded p-extension of $A F_{1}$.

Like the grounded extension, the grounded p-extension of an argumentation framework $A F$ includes the set of the elements of $A$ which are not attacked. Hence:

Lemma 4 Let $A F=\langle A, R\rangle$ be a finite argumentation framework. If $A F$ is acyclic, then the grounded p-extension of $A F$ is nonempty.

Thus, every finite argumentation framework has at least one preferred p-extension, a unique grounded p-extension and zero, one or many stable p-extensions.

Let us now introduce a notion of complete p-extension.

Definition 12 (complete p-extensions) Let $A F=\langle A, R\rangle$ be a finite argumentation framework and let $S$ be a $p$ admissible set for $A F . S$ is a complete p-extension of $A F$ if and ony if every argument which is acceptable w.r.t. $S$ and without indirect conflicts with $S$ belongs to $S$.

From the definition, it comes immediately that:

Lemma 5 A set of arguments $S$ without indirect conflicts is a complete p-extension of $A F$ if and only if $\mathcal{F}_{A F}^{p}(S)=S$.

We also have:

Lemma 6 The grounded p-extension of a finite argumentation framework $A F$ is a complete p-extension of $A F$.

While the grounded extension of an argumentation framework $A F$ is included into the intersection of all the complete extensions of $A F$, it is not the case in general that the grounded p-extension of $A F$ is included into every preferred p-extension of $A F$.

Let us now define several inference relations based on our prudent semantics for argumentation frameworks:
Definition 13 (prudent inference relations) $\sim_{p}^{q, s}$ denotes the inference relation obtained by considering a prudent semantics $s$ (where $s=P($ referred), $s=S($ table) or $s=G($ rounded $))$ and $q$ is an inference principle, either credulous $(q=\exists)$ or skeptical $(q=\forall)$.

For instance, $S \subseteq A$ is a consequence of $A F$ w.r.t. $\sim_{p}^{\forall, P}$, noted $A F \sim_{p}^{\forall, \bar{P}} S$, indicates that $S$ is included into every preferred p-extension of $A F$.

We have compared all the prudent inference relations w.r.t. cautiousness, assuming that every $A F$ under consideration has a stable p-extension. ${ }^{3}$

Lemma 7 Let AF be a finite argumentation framework. If $A F$ has a stable p-extension, then the intersection of all preferred p-extensions of $A F$ is included into the grounded p-extension of $A F$.

Based on the previous lemmata, we have obtained the following results:

Proposition 3 The cautiousness relations reported in the following table hold for every finite argumentation framework which has a stable p-extension (Each time a cell contains $a \subseteq$, it means that for every $A F=\langle A, R\rangle$ and every $S \subseteq A$, if $S$ is a consequence of $A F$ w.r.t. the inference relation indexing the row, then $S$ is a consequence of $A F$ w.r.t. the inference relation indexing the column.)

\begin{tabular}{|c|c|c|c|c|c|}
\hline & $\sim_{p}^{\exists, P}$ & $\sim_{p}^{\exists, S}$ & $\sim_{p}^{\forall, P}$ & $\sim_{p}^{\forall, S}$ & $\sim_{p}^{, G}$ \\
\hline$\sim_{p}^{\exists, P}$ & $=$ & $\nsubseteq$ & $\nsubseteq$ & $\nsubseteq$ & $\nsubseteq$ \\
$\sim_{p}^{\exists, S}$ & $\subseteq$ & $=$ & $\nsubseteq$ & $\nsubseteq$ & $\nsubseteq$ \\
$\sim_{\forall, P}^{\forall, P}$ & $\subseteq$ & $\subseteq$ & $=$ & $\subseteq$ & $\subseteq$ \\
$\sim_{p}^{\forall, S}$ & $\subseteq$ & $\subseteq$ & $\nsubseteq$ & $=$ & $\nsubseteq$ \\
$\sim_{p}^{, G}$ & $\subseteq$ & $\subseteq$ & $\nsubseteq$ & $\subseteq$ & $=$ \\
\hline
\end{tabular}

One can note that the cautiousness picture for prudent inference relations is similar to the one for the inference relations induced from Dung's semantics (assuming that the argumentation frameworks under consideration have stable extensions):

$$
\sim_{p}^{\forall, P} \subset \sim_{p}^{\forall, S} \subset \sim_{p}^{\exists, S} \subset \sim_{p}^{\exists, P} .
$$

\section{Comparisons with Dung's Framework}

Let $\sim^{q, s}$ denote the inference relation obtained by considering Dung's semantics $s$ (where $s=P$ (referred), $s=S($ table $)$ or $s=G($ rounded $))$ and $q$ is an inference principle, either credulous $(q=\exists)$ or skeptical $(q=\forall)$. We have obtained the following results:

\footnotetext{
ialize.

${ }^{3}$ When it is not the case, both inference relations $\sim_{p}^{\exists, S}$ and $\mid \sim_{p}^{\forall, S}$ triv-
} 
Proposition 4 The cautiousness relations reported in the following table hold for every finite argumentation framework which has a stable p-extension.

\begin{tabular}{|c|c|c|c|c|c|}
\hline & $\sim_{p}^{\exists, P}$ & $\sim_{p}^{\exists, S}$ & $\sim_{p}^{\forall, P}$ & $\sim_{p}^{\forall, S}$ & $\nu_{p}^{,{ }^{\prime}}$, \\
\hline & $q, \supseteq$ & $\not \subset, \supseteq$ & $\not \subset, \supseteq$ & $\not \subset, \supseteq$ & $\not \subset$, \\
\hline$\sim^{\exists,}$ & $\nsubseteq, \nsupseteq$ & $\not \subset, \supseteq$ & $\not \subset, \supseteq$ & $\nsubseteq, \supseteq$ & $\not \subset$ \\
\hline & $\subseteq, \nsupseteq$ & $\subseteq, \nsupseteq$ & $\not \subset, \supseteq$ & $\subseteq, \nsupseteq$ & $=$ \\
\hline$\sim^{\forall, S}$ & $\subseteq, \nsupseteq$ & $\subseteq, \nsupseteq$ & $\not \subset, \supseteq$ & $\subseteq, \nsupseteq$ & $\not \subset, \supseteq$ \\
\hline$\sim^{\cdot, G}$ & $\subseteq, \nsupseteq$ & $\subseteq, \nsupseteq$ & $\not \subset, \supseteq$ & $\subseteq, \nsupseteq$ & $=$ \\
\hline
\end{tabular}

In the light of the two tables, one can observe that the most cautious inference relations among those considered here is $\sim_{p}^{\forall, P}$. As expected, credulous prudent inference relations are strictly more cautious than credulous nonprudent ones. More surprisingly, $\sim_{p}^{\forall, P}$ is strictly more cautious than $\sim^{\forall, P}$.

Before concluding the paper, let us briefly consider some complexity issues related to our prudent inference relations. First of all, it is easy to show that, given a finite argumentation framework $A F$, deciding whether an argument indirectly attacks another argument and deciding whether a set of arguments is free of indirect conflict (resp. is padmissible for $A F$, is a stable p-extension of $A F$, is the grounded p-extension of $A F$ ) are in $\mathrm{P}$.

We showed in a previous paper [6] that considering sets of arguments (instead of single arguments) as input queries for the inference problem does not lead to a complexity shift (the purpose is to determine whether such sets are derivable from a given finite argumentation framework $A F$ ). As to the prudent inference relations, it comes that deciding whether a set of arguments is a preferred p-extension of $A F$ or whether it is included into all stable p-extensions of $A F$ are in coNP, and that deciding whether a set of arguments is included into a preferred p-extension (resp. a stable pextension) of $A F$ is in NP. Finally, deciding whether a set of arguments is included into all preferred $\mathrm{p}$-extensions of $A F$ is in $\Pi_{2}^{p}$. Accordingly, our prudent inference relations are not computationally more complex that the corresponding ones based on Dung's semantics (see $[8,11])$.

\section{Conclusion and Perspectives}

We have presented new prudent semantics within Dung's theory of argumentation. Under such prudent semantics, two arguments cannot belong to the same extension whenever one of them attacks indirectly the other one. This leads to a better handling of controversial arguments than in Dung's approach.

Our work calls for some perspectives. One of them consists in developing specific algorithms for computing pru- dent extensions, based on algorithms for computing extensions like those described in [5, 9].

\section{References}

[1] L. Amgoud and C. Cayrol. Inferring from inconsistency in preference-based argumentation frameworks. J. of Automated Reasoning, 29:125-169, 2002.

[2] P. Baroni and M. Giacomin. Solving semantic problems with odd-length cycles in argumentation. In ECSQARU'03, volume 2711 of LNAI, pages 440-451, 2003.

[3] P. Baroni, M. Giacomin, and G.Guida. Extending abstract argumentation systems theory. Art. Intelligence, 120(2):251270, 2000.

[4] P. Besnard and Anthony Hunter. A logic-based theory of deductive arguments. Art. Intelligence, 128:203-235, 2001.

[5] C. Cayrol, S. Doutre, and J. Mengin. On decision problems related to the preferred semantics for argumentation frameworks. J. of Logic and Computation, 13(3):377-403, 2003.

[6] S. Coste-Marquis, C. Devred, and P. Marquis. Symmetric argumentation frameworks. In ECSQARU'05, volume 3571 of LNAI, pages 317-328, 2005.

[7] Y. Dimopoulos, B. Nebel, and F. Toni. On the computional complexity of assumption-based argumentation for default reasoning. Art. Intelligence, 141:57-78, 2002.

[8] Y. Dimopoulos and A. Torres. Graph theoretical structures in logic programs and default theories. Theoretical Computer Science, 170:209-244, 1996.

[9] S. Doutre and J. Mengin. On sceptical vs credulous acceptance for abstract argument systems. In NMR'04, pages 134 139, 2004.

[10] P. M. Dung. On the acceptability of arguments and its fundamental role in nonmonotonic reasoning, logic programming and n-person games. Art. Intelligence, 77(2):321-358, 1995.

[11] P. Dunne and T. Bench-Capon. Coherence in finite argument system. Art. Intelligence, 141:187-203, 2002.

[12] M. Elvang-Gøransson and A. Hunter. Argumentative logics: Reasoning with classically inconsistent information. Data and Knowledge Engineering, 16:125-145, 1995.

[13] M. Elvang-Gøransson, J. Fox, and P. Krause. Acceptability of arguments as logical uncertainty. In ECSQARU'93, volume 747 of $L N C S$, pages 85-90, 1993.

[14] J. Pollock. How to reason defeasibly. Art. Intelligence, 57(1):1-42, 1992.

[15] A. Prakken and G. Vreeswijk. Logics for defeasible argumentation. volume 4 of Handbook of Philosophical Logic, $2^{\text {nd }}$ edition, pages 219-318. Kluwer Academic Publishers, 2002.

[16] G. Simari and R. Loui. A mathematical treatment of defeasible reasoning and its implementation. Art. Intelligence, 53(2-3):125-157, 1992.

[17] S. Toulmin. The Uses of Argument. Cambridge University Press, 1958. 